\title{
Epigenetics and Transcriptomics to Detect Adverse Drug Effects in Model Systems of Human Development
}

\author{
Nina V. Balmer and Marcel Leist \\ Doerenkamp Zbinden Chair for In Vitro Toxicology and Biomedicine, University of Konstanz, Konstanz, Germany
}

\begin{abstract}
Prenatal exposure to environmental chemicals or drugs has been associated with functional or structural deficits and the development of diseases in later life. For example, developmental neurotoxicity (DNT) is triggered by lead, and this com pound may predispose to neurodegenerative diseases in later life. The molecular memory for such late consequences of early exposure is not known, but epigenetic mechanisms (modification of the chromatin structure) could take this role. Examples and underlying mechanisms have been compiled here for the field of DNT. Moreover, we addressed the question as to what readout is suitable for addressing drug memory effects. We summarize how complex developmental processes can be modelled in vitro by using the differentiation of human stem cells. Although cellular models can never replicate the final human DNT phenotype, they can model the adverse effect that a chemical has on key biological processes essential for organ formation and function. Highly information rich transcriptomics data may inform on these changes and form the bridge from in vitro models to human prediction. We compiled data showing that transcriptome analysis can indicate toxicity patterns of drugs. A crucial question to be answered in our systems is when and how transcriptome changes indicate adversity (as opposed to transient adaptive responses), and how drug induced changes are perpetuated over time even after washout of the drug. We present evidence for the hypothesis that changes in the histone methylation pattern could represent the persistence detector of an early insult that is transformed to an adverse effect at later time points in life.
\end{abstract}

Developmental toxicity (DT) testing is one of the technically most challenging fields of toxicology. There is a huge demand for more cost effective, faster and more accurate assays [1]. DT may be caused by chemicals, drugs, pesticides and other compounds that interfere with biological processes essential for reproduction. The hazard posed by reproductive toxicants is therefore a matter of great societal concern, and measures to avoid DT are highly demanding in resources, animals and time [2].

Evaluation of a single compound for DT can require hun dreds to several thousand animals. More than 1000 rats/rabbits alone are required for a single study (OECD test guideline TG426 'developmental neurotoxicity') that examines distur bances of the development of the nervous system (the focus of the current review). One problem of animal experiments in the whole area of DT is that there may be large species differ ences, and extrapolation to man can therefore fail. For instance, thalidomide effects are hard to detect in rats, while they are very pronounced in man [3] (and references therein). Strong species differences can also be observed in cell culture test systems, and therefore human cell based test systems appear most promising [4,5]. Animal studies assessing devel opmetal neurotoxicity (DNT) usually do not yield any mecha

Author for correspondence: Marcel Leist, University of Konstanz, Box 657, D 78457 Konstanz, Germany (fax +49 7531885039 , e mail marcel.leist@uni konstanz.de). nistic information or biological background data that would facilitate the extrapolation to man. This type of testing often only detects toxicity through indirect measures such as chan ged numbers of embryos per litter, altered foetal weight or the development of behavioural abnormalities. To significantly reduce the use of animals, to get further mechanistic insights and to obtain data that can be used to predict hazard to man, in vitro systems modelling critical steps of the human foetal development are being explored as alternatives [6,7]. The development of initial germ layers from pluripotent cells and the specification of organ systems, such as the central nervous system (CNS), are such critical processes, and their distur bance would be a key event in DT. The CNS is considered to be one of the most frequent targets of systemic toxicity, with the developing nervous system being particularly susceptible $[1,8]$. This susceptibility to DNT is due to a finely orches trated sequence of complex biological processes, such as proliferation, migration, apoptosis, differentiation, patterning, neurite outgrowth, synaptogenesis, myelination and neuro transmitter synthesis, which are all targets of numerous toxic chemicals [9]. DNT describes effects of chemicals on the developing nervous system. Such exposure during neurodevel opment can result in neurobehavioural alterations and severe malformations $[1,10]$. Despite its high relevance, DNT is one of the least studied forms of toxicity $[9,11]$. It is also particu larly difficult to study for three reasons: (i) DNT affects a tis 
sue that is itself changing during the experiment, also under control conditions. (ii) DNT effects may have a time offset, that is, neurofunctional deficits may be measurable only many years after the exposure to the chemical has ceased. (iii) DNT is not necessarily caused by cell death, but in most cases by changes in neuronal function, connectivity, composition or positioning [10].

Foetal exposure to some compounds may influence severity of and susceptibility to psychiatric disorders, like autism and schizophrenia, in later life $[12,13]$. Human data on DNT are only accessible from epidemiological studies, in which cause effect relationships are hard to find. As also data from rodents may lack sensitivity or predictivity [14,15], DNT hazard might be better predictable if based on biological modes of action, which can be identified using human cellular model systems.

Over the last decades, evidence has been accumulating that altered epigenetic control of gene expression is not only a cause for diseases (e.g. carcinogenesis), but also that compounds inter fering with this epigenetic control have been suspected to cause developmental defects in later life [16]. Thus, epigenetics poten tially provides a mechanistic background for DT and late onset diseases like Alzheimer's disease or Parkinson [17,18]. To allow better hazard assessment of chemicals, human relevant test systems need to be developed that allow the investigation of the long term effects of chemicals [9]. Such test systems have recently been used to study how epigenetic mechanisms could mediate the transition from an early insult by a chemical to an adverse effect on the developing nervous system $[19,20]$.

\section{Environmental Chemicals and Disease}

Due to the complexity of neural development, it is not surpris ing that the developing brain is particularly susceptible to adverse effects. For several reasons, the developing brain can be more susceptible to toxic insults than the adult brain: the placenta, although offering some protection, is not an effective barrier against environmental pollutants [21]; the blood brain barrier is incomplete up until 6 months after birth [22] and detoxification of exogenous compounds is reduced, for exam ple, by different expression pattern of detoxifying CYP enzymes [23]. Thus, DNT can occur at doses lower than those leading to other malformations [24].

It is estimated that $312 \%$ of children in the United States suffer from at least one mental disorder [24]. The most com mon neurodevelopmental disorders include motor and mental retardation, leaming disabilities and attention deficit hyperac tivity disorder [24]. It has been claimed that $3 \%$ of the devel opmental disabilities are directly caused by environmental exposure, and that another $25 \%$ are caused by interactions between genetic susceptibility and environmental factors [25]. Examples of such mental disorders related to an interaction of genetic susceptibility and environmental exposure are schizo phrenia [12] and autism [13].

The Barker hypothesis: developmental origins of late disease. Studies on birth cohorts by David Barker showed that low birth weight or small head circumference of infants is associated with an increased risk of developing diseases like insulin resistance or coronary heart disease. The concept that parameters of foetal, infant and childhood growth can predict diseases in later life is now known as the Barker hypothesis [26]. It has been extended to encompass brain development and its disturbance by toxic chemicals $[27,28]$. This hypothesis builds on data describing the adverse effects that lead, methyl mercury and polychlorinated biphenyls had on the developing brain, and on studies that revealed associations between early life exposure to pesticides and Parkinson's disease (PD) [27]. The latter serves as a good example to explain the neurodevel opmental Barker hypothesis (fig. 1). Symptoms of PD occur when the number of dopaminergic neurons in the substantia nigra of the brain decreases below approximately $25 \%$ of the normal amount. During normal ageing, the number of dopami nergic neurons decreases but does not cross the threshold below which PD symptoms arise. Genetic disposition to PD leads to a faster decline in the number of dopaminergic neu rons. The hypothesis of a 'neurodevelopmental origin of PD' suggests that a toxic insult during neurodevelopment leads to a lower number of dopaminergic neurons early in life. This does not cause any PD symptoms early, but the normal life long decrease in dopaminergic neurons would lead to an early onset of PD (fig. 1). Some evidence indicates that environ mental toxicants can indeed affect dopaminergic neurons in early life stages: In developing embryonic stem cells, the envi ronmental toxicant methyl mercury has been shown to selec tively impair the formation of dopaminergic neurons [29] and several compounds affect the neurite growth of developing dopaminergic neurons $[30,31]$.

There are many more examples of developmental exposure to toxicants contributing to diseases in later life $[32,33]$. For example, developmental exposure to lead $(\mathrm{Pb})$ has recently been found to contribute to late onset Alzheimer's disease [17]. Also, exposure of mice to the environmental pollutant

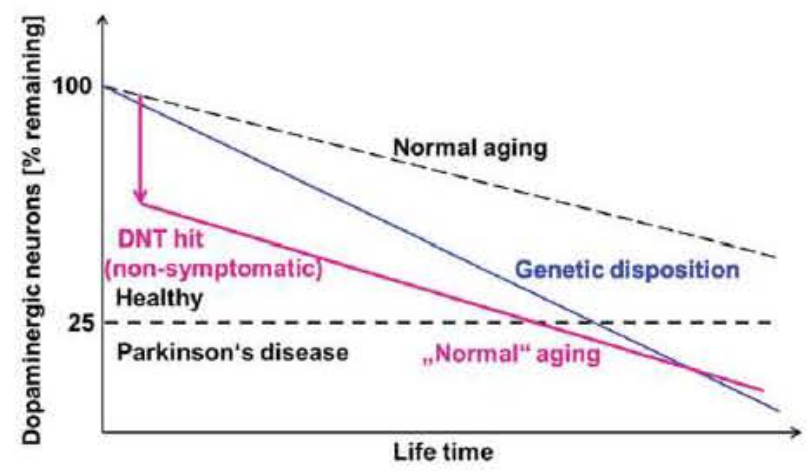

Fig. 1. Long term consequences of early life insults. This is shown on the example of dopaminergic neurodegeneration in Parkinson's disease (PD). PD occurs when the number of dopaminergic neurons declines below a certain threshold. This can be caused by genetic disposition (blue). Exposure to a developmental neurotoxicity (DNT) compound during neurodevelopment may decrease the number of dopaminergic neurons in the brain, but does not result in immediate symptoms (arrow). The normal age dependent decline in the number of dopami nergic neurons can then lead to the onset of PD. Graphics inspired by [27]. 
methyl mercury $(\mathrm{MeHg})$ during pregnancy has been shown to disturb leaming and to cause a predisposition to depressive behaviour in male offspring [34].

These are examples of environmental factors triggering dis eases in later life, sometimes even in subsequent generations. When investigating how subsequent generations are affected by early developmental insults, two issues need to be consid ered. Firstly, it is important to separate the effects that a chem ical has on the mother from the effects it has on her offspring. Secondly, one has to bear in mind that there are several levels of multi generational and transgenerational effects. In contrast to multi generational effects, transgenerational effects are transmitted between generations but do not involve direct exposure. For that, germ line transmission needs to be involved. The generation showing the effects should not have been exposed to the chemical directly. For in utero exposure, F3 generation is the first generation that is not directly exposed to the chemical (fig. 2A). In post natal exposure sce narios, the first not directly exposed generation is the F2 gen eration (fig. 2B).

Real transgenerational effects have been shown for the endocrine disruptor vinclozolin. Exposure to vinclozolin dur ing embryonic gonadal sex determination (fig. 2A) was shown to induce adult onset disease for multiple generations, includ ing F3, which is the first generation not directly exposed after in utero exposure [35]. There is growing evidence that trans generational effects of toxicants are mediated by epigenetic mechanisms $[36,37]$.

\section{Epigenetics and Disease}

Epigenetics describes the alterations of gene expression due to structural changes of chromatin. There are several mechanisms which can determine the chromatin structure and the access of transcription factors to their regulatory sequence, which further on will be called epigenetic mechanisms. Their balance is interdependent and essential for normal development and cel lular function. There are five categories of epigenetic mecha nisms known to affect chromatin structure: biochemical

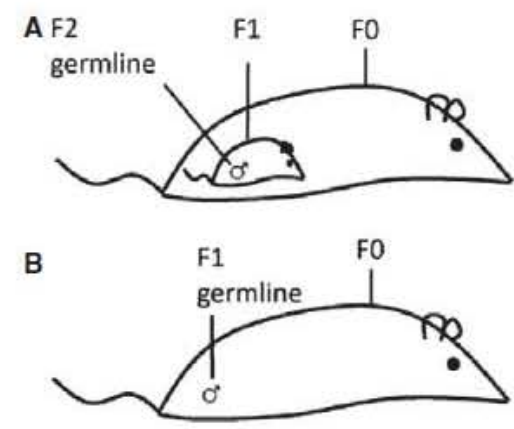

Fig. 2. Direct exposure of generations. (A) in utero exposure: Gestat ing female (FO) is exposed to the chemical. Therefore, the offspring is directly exposed via the umbilical blood (F1). The F2 generation is directly exposed as germ line of F1. (B) post natal or adult exposure: Post natal or adult individual is exposed to the chemical (F0). The F1 generation is directly exposed as germ line of FO. modifications of DNA, post translational modifications (PTM) of histones, several classes of non coding RNAs, chromatin remodelling complexes which are ATP dependent protein complexes that perform nucleosomal sliding, and the exchange of histone variants which influence the regional chromatin condensation [38]. Biochemical 'modifications of DNA' have in the past been regarded as consisting mainly of cytosine methylations at $\mathrm{CpG}$ sites. It has become increasingly clear that DNA methylation can follow more complex patterns. Moreover, higher oxidation states of 5 methylcytosine (i.e. hydroxymethylation and formylation) have been found to be biologically relevant, and a group of responsible oxidoreducta ses (TET1 3) has been identified. Also adenine methylation can occur, and this widening array of DNA modifications may reflect variations in gene expression [39].

Post translational modifications (PTM) of histones are known to be one of the key mechanisms for regulating proper gene expression [40]. It has been debated whether the struc ture and interactions by net charge of the amino tails of hi stones define the structure and function of chromatin [41] or whether a so called histone code exists. The latter describes the hypothesis that distinct histone amino terminal modifica tions are responsible for interaction of chromatin associated proteins and therefore dictate transcriptionally active and silent chromatin states [42]. Taken together, it seems likely that both, net charge effects and specific binding to modifications, are the basis for regulating chromatin structure and gene expression.

There are histone marks that have been clearly associated with one chromatin state (fig. 3). A histone modification, known to be associated with the open, transcriptionally active chromatin (euchromatin) is the methylation of histone $\mathrm{H} 3$ at the lysine in position 4 (H3K4me3). The chromatin can also be opened by acetylation (Ac) of lysine 9 of H3 (H3K9Ac). Other modifications like $\mathrm{H} 3 \mathrm{~K} 27 \mathrm{me}$ and $\mathrm{H} 3 \mathrm{~K} 9 \mathrm{me}$ correlate with transcriptionally silenced chromatin (heterochromatin). Unfortunately, the exclusiveness of histone marks to one kind of chromatin is in most cases not as clear as for H3K4me [43].

Due to the multitude of enzymes and cofactors that regulate epigenetic modifications, it seems likely that chemicals are able to disturb this finely tuned regulatory network at multiple levels [44]. Firstly, chemicals can influence the activity of the epigenetic modifiers. Secondly, toxicants can alter gene expression levels of the epigenetic modifiers or, thirdly, can be in general geno toxic and induce mutations in the genes of the epigenetic modifiers which leads to miss regulation or function [40]. Changes in gene expression levels of epigenetic modifiers can be tested by standard transcriptomics methods [45]. However, to obtain information on altered activities of chromatin modifying enzymes, methods to quantify changes of histone PTMs or DNA methylation levels are necessary. Toxicological test systems evaluating such end points are hard to establish, although it is well known that certain classes of pharmaceuticals (e.g. HDAC inhibitors) do modify epigenetic marks $[19,44,46,47]$. Also, it is known that exposure to sev eral environmental chemicals and other stressors can result in 
Euchromatic marks

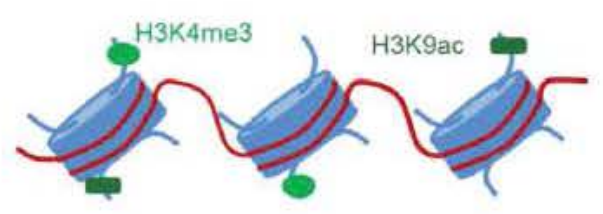

Heterochromatic marks

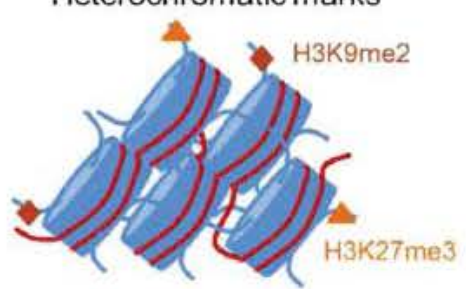

Fig. 3. Post translational modifications (PTM) characteristic for Eu or Heterochromatin. Blue lines represent histone tails. Green and orange object represent PTM of histones. See text.

altered epigenetic marks. For this reason, it appears highly important to establish new test systems that evaluate epige netic changes and to incorporate epigenetic end points into already existing test systems.

Diseases and toxicity caused by epigenetic mechanisms.

Studies on monozygotic twins provide an elegant model to study the environmental influence on gene expression. Using this model, it has been shown that twins at 50 years of age in contrast to 3 year old twins differ in the DNA methylation pattem and the $\mathrm{H} 3$ and $\mathrm{H} 4$ acetylation pattern of their lympho cytes [48]. It is likely that these changes are not only caused by nutrition, but also by exposure to environmental chemicals and drugs.

Mental retardation or autism, which can be caused by dis turbance of neurodevelopment, affects about $3 \%$ of children. Many genes associated with these diseases affect epigenetic mechanisms [49 51]. One example of such an epigenetically caused mental retardation disorder is the Rett syndrome. It is caused by mutations in the gene encoding MeCP2 which binds to methylated $\mathrm{CpG}$ islands [52]. One of MeCP2's func tions is transcriptional silencing by recruiting HDACs.

Changes in DNA methylation have been shown to lead to altered expression of imprinted genes. Several diseases (Prad er Willi, Wilms tumour, Angelmann syndrome) are based on the failure of imprinting [53].

Intriguingly, many late onset disorders and altered behav iours that are caused early in life or even in the generations before (see Barker hypothesis) are associated with epigenetic alterations. A famous example is the stress response of mice that is altered dependent on maternal care. Offspring that received less maternal care are more fearful and show stronger activation of the hypothalamic pituitary adrenal (HPA) axis in response to stress. This has been associated with altered his tone acetylation levels in the glucocorticoid receptor (GR) gene promoter in the hippocampus [54]. These findings could be translated to human beings. Childhood abuse was found to alter HPA stress responses and increase the risk of suicide. This correlated with epigenetic differences in a neuron specific glucocorticoid receptor promoter in post mortem hippocampus of suicide victims [55]. The methylation of the promoter of this glucocorticoid receptor was also shown to be altered in adolescent children whose mothers were exposed to intimate partner violence during pregnancy [56]. However, it is hard to correlate such epigenetic alterations to their primary cause.
Also, evidence arises that environmental chemicals can induce many of the sporadic forms of neurological disorders and that this could be due to an altered epigenetic state. For example, the before mentioned adverse effect on learning and mood of offspring of mice treated with $\mathrm{MeHg}$ has been correlated to increased levels of DNA methylation and increased levels of $\mathrm{H} 3 \mathrm{~K} 27 \mathrm{me} 3$ and reduced $\mathrm{H} 3 \mathrm{~K} 9 / \mathrm{H} 3 \mathrm{~K} 14$ acetylation at the BDNF promoter [57]. Alzheimer's disease (AD), as mentioned earlier, may be caused by infantile exposure to lead $(\mathrm{Pb})$. Examination of such an effect in aged monkeys uncovered decreased DNA methyltransferase activ ity, which lead the authors to suggest that $\mathrm{Pb}$ exposure alters the DNA methylation pattern in early life and thereby influ ences the expression of $\mathrm{AD}$ related genes in later life [18]. The down regulation of DNA methylating enzymes and up regulation of $\mathrm{AD}$ related genes after $\mathrm{Pb}$ exposure was reproduced in the same group in human neuronal cells in vitro [17]. Accordingly, cognitive function has been shown to be affected by epigenetic drugs that alter histone acetyla tion $[58,59]$.

Exposure to some chemicals can even lead to altered behav iour and an increased incidence of diseases three generations after the exposure. This is best studied for endocrine disrup tors. Exposure to bisphenol A during early development has been shown to alter DNA methylation in the foetal mouse forebrain and to alter behaviour [36]. Embryonic exposure to the endocrine disruptor vinclozolin has been shown to lead to adult onset diseases, including for example prostate disease, testis and immune system abnormalities in the first generation, which persisted for four subsequent generations [35]. This was due to altered DNA methylation pattern in the male germ line and resulted also in heritable changes in gene expression in the brain, even two generations later (F3). These altered expression patterns correlated with sex specific altered anxi ety like behaviours up to the $\mathrm{F} 3$ generation [60]. The vincloz olin exposure three generations earlier even altered the murine response to chronic restraint stress [61].

The involvement of epigenetic mechanisms in the adverse effect of environmental chemicals has lead to the suggestion to include epigenetic modifications as end points for risk assessment [44]. Considering the severity of disorders that can be caused by environmental chemicals causing epigenetic changes, the altered epigenetic modifications seem to be good candidates to indicate health hazard. Additionally, epigenetic biomarkers could be assessed to find out if ancestral or early 
life environmental exposures occurred that are associated with adult onset disease.

\section{Modelling Biological Processes of Neurodevelopment In Vitro}

To describe the chain of events that links a chemical to the hazard it poses to the organism, different concepts have been developed. They differ in their main perspective and the intended use. For instance, the concept of 'pathways of toxicity' focuses mainly on the network of cellular regulations that deci des the eventual cell fate [62]. The concept of 'adverse outcome pathways' relates a molecular initiating event that is triggered by a chemical or its metabolite to a defined adverse outcome (http://www.oecd.org/env/ehs/testing/49963554.pdf). The con cept of 'biomarkers of toxicity' mainly deals with measurable end points that can be applied to model systems [63]. The newly proposed concept of 'toxicity endophenotypes' focuses on bio logical processes that can be modelled by in vitro systems in contrast to final phenotypes, like mental retardation, which, in most cases, can hardly be directly assessed [9]. For instance, neurodevelopment is very complex and needs many biological processes to be orchestrated correctly in time and space. Inter ference with these processes can lead to developmental neuro toxicity (DNT). The adverse outcome of DNT comprise reduced IQ, attention deficit, various sensory disturbances and others. These functionally defined phenotypes can, at present, not be modelled in in vitro systems. To develop relevant in vitro model systems for DNT, the final phenotype needs to be linked to effects that the DNT causing chemical has in in vitro systems [1]. Therefore, we need to understand how the final phenotype is linked to the altered biological state of the nervous system in vivo, also called the endophenotype, and how the phenotype is linked to basic biological processes that are affected by the chemical in vivo (fig. 4). Additionally, we need to understand the link of the endophenotype to the basic biological processes affected by the chemical, not only in vivo but also in vitro. Thus, the toxicity endophenotype (TEP) describes the biologically quantifiable altered functionality of parts of the nervous system due to exposure to a DNT chemical. This is triggered by a chemical and can be caused by several biological processes dis turbed by the chemical. Those biological processes then can be tested in vitro. Distinct biological processes affected by a chemical causing DNT can be modelled in several test systems $[1,5,9,64]$.

For instance, it is known that interference with neurite out growth (biological process) can lead to altered electrical cir cuits (toxicity endophenotype), which has been associated with schizophrenia and mental retardation (DNT effect) [64 71].

In vitro test systems can model all important biological neu rodevelopmental processes that in turn could relate to toxicity endophenotypes (TEPs). TEPs describe the biologically quan tifiable altered functionality of parts of the nervous system due to exposure to a DNT chemical. They can be regarded as a link between basic biological processes that are disturbed by a DNT compound and the final DNT phenotype described by clinical end points (fig. 4).

Another important point to consider in DNT hazard assess ment is that data derived from animal studies are often not pre dictive for human hazard [72]. Thus, human based test systems have to be developed and integrated to investigate the effects of chemicals on biological processes. The results might then enable the examination of their effect on the TEP and the DNT outcome. This will allow better prediction of DNT hazard.

Transcriptional profiling has been proposed as sensitive end point to distinguish neural differentiation states during normal and disturbed development $[10,63,73$ 75]. Marker genes speci fying time and region of the differentiating cells can be assessed by transcriptional profiling and it has been shown that such mar ker gene expression during neural differentiation of embryonic stem cells occurs in a similar manner to in vivo [76]. However, it is important to note that changes in marker gene expression in a test system, for example, of neurally differentiated hESC, can have two different reasons. Firstly, the changes can be caused by the acute effect of the compound on a biological process, thereby possibly representing a pathway of toxicity or an adverse outcome pathway. Secondly, the changes can be caused by an altered differentiation and therefore have to be regarded as a fingerprint of the culture. The latter would then represent the above mentioned toxicity endophenotype for DNT.

\section{Disturbed Neural Patterning Indicated by an Altered Transcriptomics Fingerprint}

A better understanding of the mode of action by which com pounds interfere with biological processes linked to DNT is

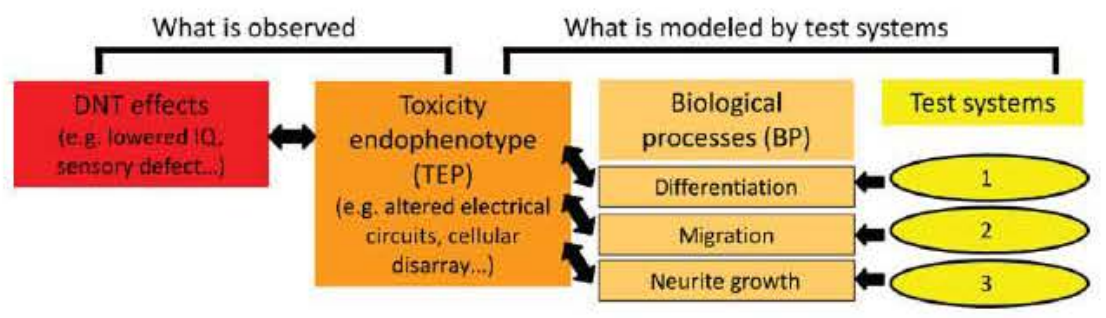

Fig. 4. Integrated test systems to investigate toxicity endophenotypes related to the developmental neurotoxicity (DNT) outcome. A DNT effect (red), observed in human beings, is linked to a toxicity endophenotype (TEP) (orange). The TEP describes the biologically quantifiable altered functionality of parts of the nervous system that is triggered by a DNT chemical and that is most likely due to an anatomically or functionally altered connectivity. This can be caused by disturbance of several biological processes (yellow). Test systems can model these biological processes and can be used to investigate the modes of actions by which chemicals disturb the biological processes. 
important to better identify and assess their DNT hazard. For example, the disturbance of migration or patteming, which are biological processes, can lead to neural tube defects $[19,77,78]$. For correct patteming, specific cell types need to be formed at the correct time in the differentiating brain. Tran scriptome analysis was found to be a suitable approach to assess alterations in the patterning of neural differentiation [63]. It allows a comprehensive description of overall changes of the cell culture during differentiation [75,79 81]. Global alterations in the cell culture's transcriptomic fingerprint were found to indicate altered neural development $[19,78]$. A trans criptomics fingerprint also means that specific marker genes are present or absent in specific cell types $[10,19,82]$. Such marker genes have been used to look at specific changes in the neural differentiation of hESC, e.g. in our laboratory. For example hESC express typical genes like OCT4, NANOG or DNMT3B (indicated in fig. 5 as A, B and C). Neuroepithelial precursor cells (NEP) express neural precursor genes like PAX6, OTX2 and FOXG1 (indicated in fig. 5 as D, E and F). Neural differentiation does, in practice, not result in a pure population of NEPs. During differentiation from hESC to NEP, intermediate cellular populations occur that may still express some hESC marker genes, already express some NEP marker genes and also express marker genes that specifically arise in these intermediate stage cells (e.g. ZIC3 indicated as M in fig. 5). Early short exposure to a DNT toxicant might slightly alter the transcriptomic fingerprint of the cellular pop ulation at early time points of differentiation (fig. 5 right panel, upper part). If this toxic insult is removed early enough, the system might be able to compensate for the early disturbance and still give rise to a normal population of NEPs, indicating normal neural development. If the exposure to the DNT compound is prolonged, the cellular differentiation is altered; that is indicated by an altered transcriptomic finger print (X, Y and $\mathrm{Z}$ in fig. 5). In this case, the neural patterning is changed and the population contains different cell types. Accordingly, many genes are changed. Also, mixed cellular populations can occur, still expressing some stem cell or inter mediate stage marker genes. Identification of altered develop ment can be done by comparison of normal NEP to the treated population (blue boxes in fig. 5) [19].

Choosing few marker genes to screen for DNT chemicals poses the risk to identify false negative chemicals. Transcripto mics analysis is useful to identify genes highly regulated during specific differentiation periods [76]. Using this knowledge, the marker gene panel can be broadened. Marker genes can also be chosen according to their role in neural development and in neural tube defects. For example, marker genes that play a role in neural tube defects and are highly regulated during neural differentiation could be chosen (Emx2 and Zic3). However, also marker genes that are absent under normal conditions, for example, markers for other lineages like $T$ (brachyury) for mesodermal lineage or Sox 7 for endodermal lineage, have been investigated and need to be further developed [19].

\section{The Role of Epigenetics for Time-Dependent Effects of Chemicals on Development}

Environmental chemicals as well as drugs can influence the epigenome $[27,57,83]$, but it is not clear how this affects

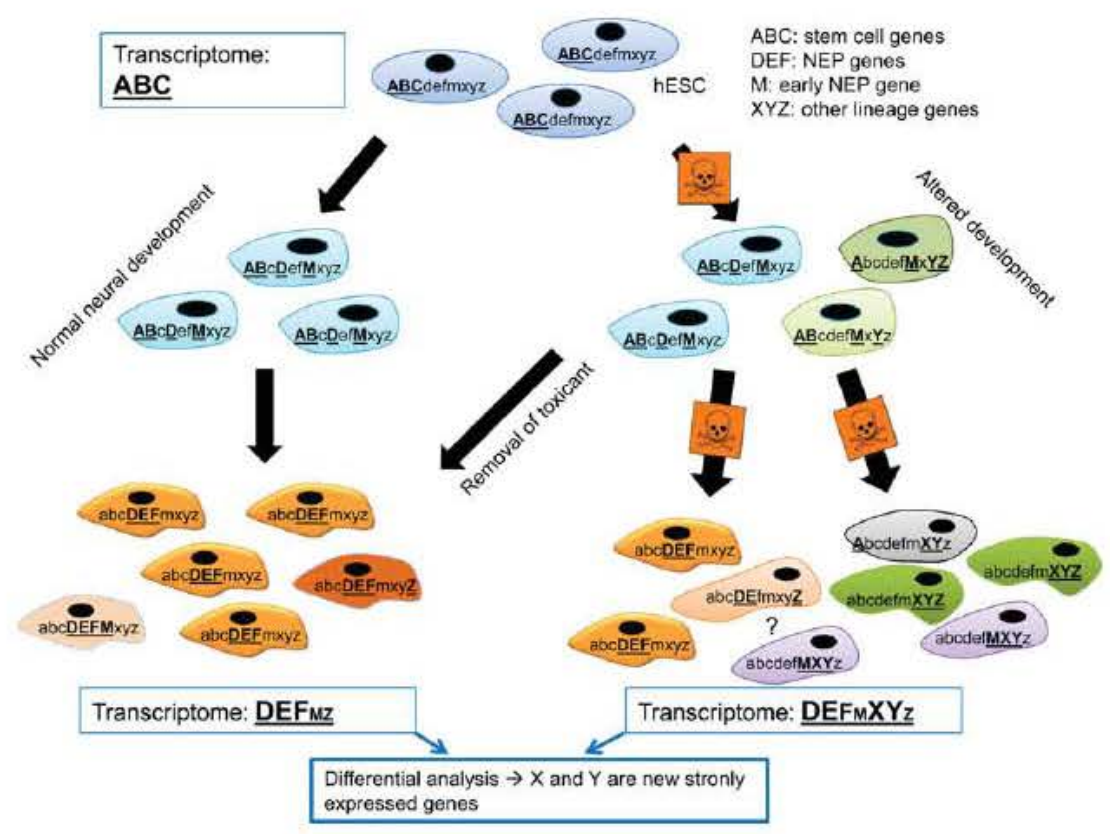

Fig. 5. Simplified overview on how normal neural differentiation can be disturbed by developmental neurotoxicity (DNT) compounds. hESC (dark blue) expressing phenotypic marker genes (indicated as A, B, C) can differentiate to neuroepithelial precursor cells (NEP) (orange) expressing neu ral marker genes (indicated by D, E, F). Intermediate cellular phenotypes occur during the differentiation process. They express some hESC marker genes, some intermediate genes (M) and some neural marker genes. Eventually, altered intermediate cells caused by short exposure to a DNT com pound followed by its removal can be compensated for by the system. Prolonged exposure can lead to altered neural development. 


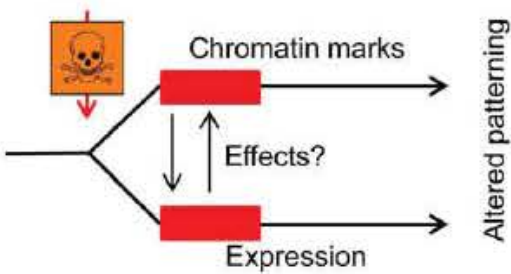

Simultaneous hit

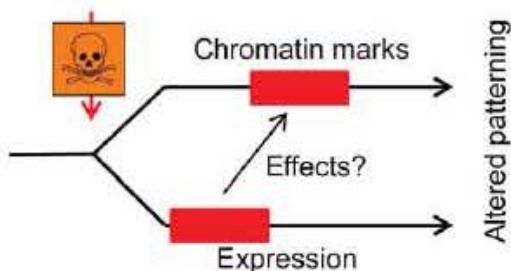

Expression first altered

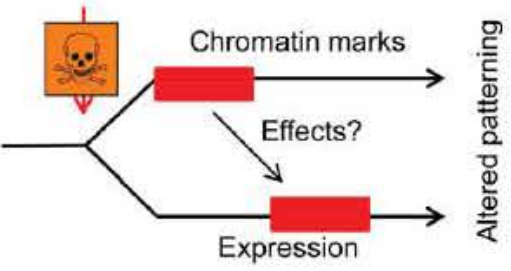

Chromatin altered first

Fig. 6. Question of cause and consequence in disturbed neural development and altered epigenetic marks. Altered patterning indicated by an altered expression pattern (see fig. 5) is associated with altered chromatin marks (histone methylation patterns at the promoters of marker genes). Altera tions in the transcriptome and in chromatin marks can be caused in three ways. Firstly, the marks can be altered simultaneously by toxicant inter ference with both mechanisms. Secondly, altered gene expression can lead to a different cellular fate, having different chromatin marks. Thirdly, the toxicant can alter the chromatin marks which leads to alteration of gene expression, finally leading to altered differentiation.

A

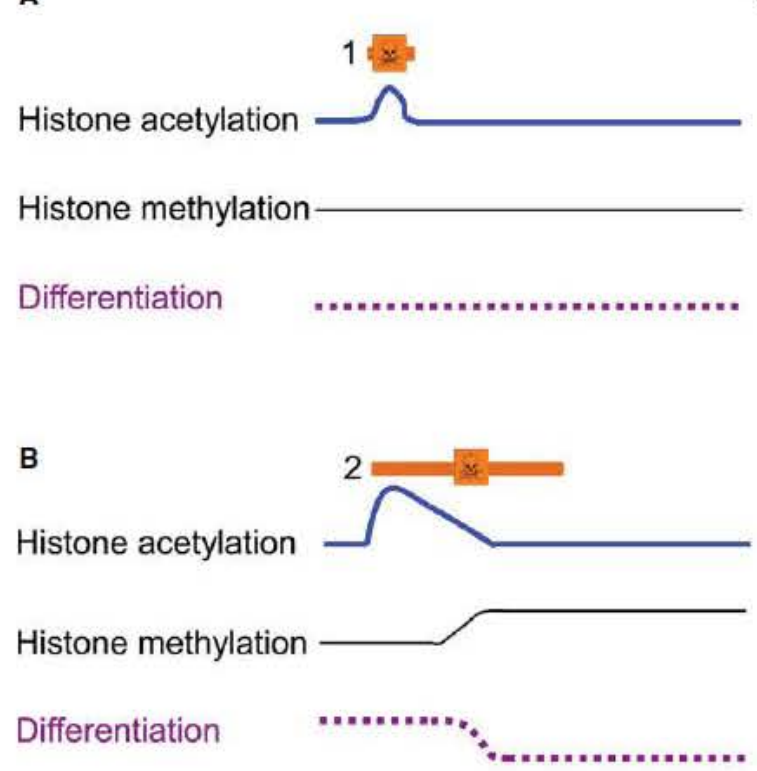

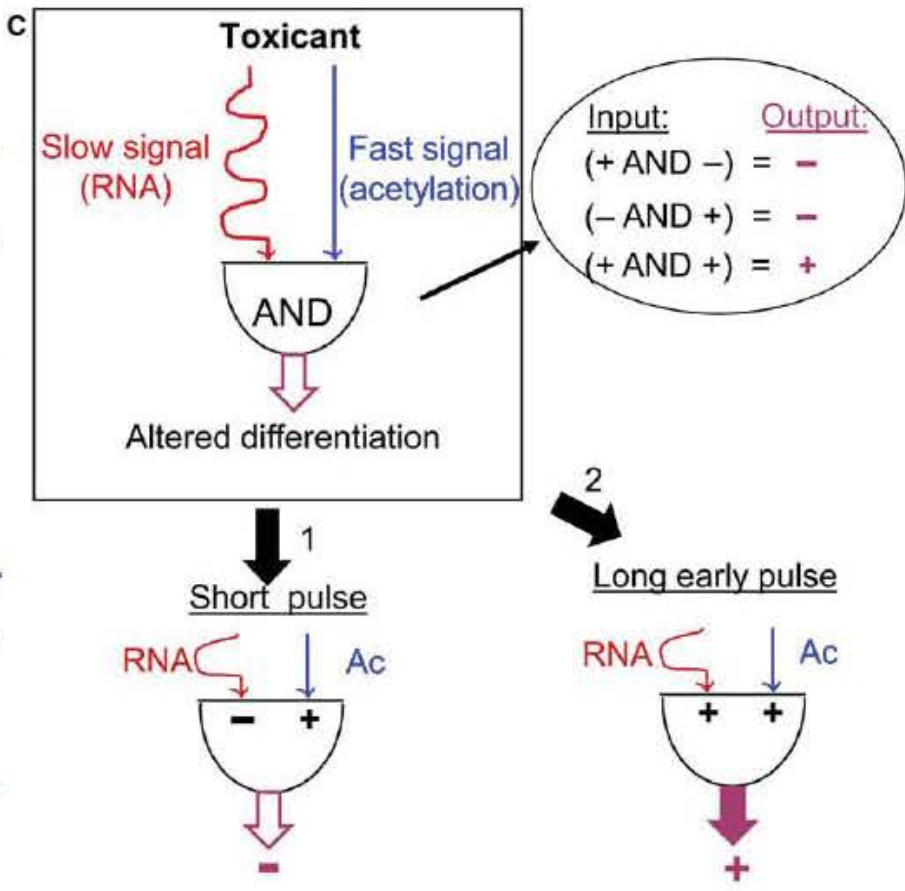

Fig. 7. Epigenetic changes as persistence detector of toxic insults. (A) Short early exposure to a valproic acid (VPA) or trichostatin A (TSA) tran siently increases histone acetylation but does not alter the histone methylation pattern or the differentiation track. (B) Increased early exposure to VPA or TSA also transiently increases acetylation levels. Subsequently, histone methylation patterns are changed, and neural differentiation is dis turbed. (C) Exposure to a toxicant might fast alter acetylation and seems to alter the transcriptomic fingerprint in a slower manner. Only if both changes occur (input + and + ), the signal seems to be mediated towards an altered differentiation track (output purple +). (1) Altered acetylation without altered transcriptome does not seem to affect histone methylation and differentiation. (2) Altered acetylation plus altered transcriptome change histone methylation and differentiation.

transcription and development. A major question is for instance, whether epigenetic changes are cause or consequence of transcriptome changes.

An altered histone methylation pattern could trigger altered expression or could be secondary due to an altered transcrip tion/differentiation (fig. 6). To investigate which explanation is more likely, time dependent data need to be gathered.

A model system bases on the differentiation of human embryonic stem cells to neuroepithelial precursor cells (NEP) has been developed in our laboratory to address the question how drugs interfere with neural development dependent on the exposure period, and what role epigenetic changes play in this [19]. The histone deacetylase inhibitors (HDACi) trichostatin A (TSA) and valproic acid (VPA), which are also known DNT compounds, altered the transcriptome of the NEP after prolonged exposure. However, the primary effect of the HDACi (increase in histone acetylation levels) was transient and only present for about 2 days [19].

Short term exposure ( $<2$ days) did not result in an altered transcriptome of the final NEP population. Hundreds of genes were altered by short term exposure, but they all returned to normal levels. In contrast to this, longer exposure ( $>4$ days) 
resulted in a permanently disturbed development, also when the drug was washed out later. Thus, exposure to DNT com pounds can alter neural differentiation depending on the expo sure period; only prolonged exposure leads to an adverse effect [19]. Epigenetic mechanisms could be the persistence detector that decides whether exposure to a chemical is long enough to lead to an adverse outcome [84,85]. Epigenetic changes might need to accumulate until a critical threshold is reached. Obviously, a hit and run model, in which a short chemical exposure is sufficient to trigger all downstream con sequences, did not apply to DNT effects. From this, the ques tion arises how prolonged exposure is measured in a cell. What could be the molecular basis of a persistence detector?

For example, short exposure to drugs can increase histone acetylation levels in our test system transiently without alter ing the overall differentiation or the histone methylation pat tern at the promoters of marker genes (fig. 7A). In contrast to this, prolonged exposure increased histone acetylation levels, but additionally altered histone methylation patterns and neural differentiation (fig. 7B). This could indicate that changes in histone methylation patterns could represent the persistence detector for toxicant exposure, and the differentiation track is disturbed only if this pattern is changed. We postulate that two changes are necessary after toxicant exposure to alter his tone methylation patterns and the neural differentiation of hESC: altered histone acetylation levels, which can happen very fast and transient, and changes in the transcriptome, which occur slower (fig. 7C). The output is affected (+) only if both effects occur $(+)$. In summary, it seems as if alterations in histone methylation patterns of the promoters of marker genes accumulate during prolonged exposure and correlate with transcriptome alterations. Nonetheless, possibly also other epigenetic changes may be revealed upon further evaluation, for example, changes in biochemical modifications of DNA, given the interconnectedness of all forms of epigenetic modifi cations.

\section{Conclusion}

We have summarized here that transcriptomic changes of dif ferentiating stem cells can define changes of the neural differ entiation. Such altered differentiation has been found to be accompanied by changes in the histone methylation patterns in promoters of marker genes of the neural differentiation. These changes in histone methylation patterns occurred after pro longed, but not short termed early drug exposure. They may represent the underlying mechanism of altered differentiation. Epigenetic mechanisms could determine the irreversibility of a drug effect. Prolonged chemical exposure may lead to toxicity (DNT) because epigenetic changes accumulated during expo sure to drugs and make the downstream changes irreversible.

It has been proposed that late onset disorders could be caused by early drug exposure in the form of small insults to developing cells. These may not be particularly conspicuous at the time of drug exposure, but the subtle changes may be 'memorized' by the cells. This 'memory effect', that is, the perpetuation of non lethal damage over time, could also be mediated by epigenetic changes. If such epigenetic changes occur in the germ line, a predisposition even of the F3 genera tion to certain diseases could be explained. Thus, epigenetic changes may represent memory effects in DT in which the drug's primary insult is stably maintained over time and can cause large phenotypic changes and altered transcriptomes at later time points in life. Such changes may not only contribute to malformations, but they may also predispose to neuropsy chiatric disease or even to late age neurodegenerative diseases, especially if further insults occur, for example, due to age related processes.

\section{Acknowledgements}

We are indebted to Tanja Waldmann, Stefanie Klima, Matth ias Weng, Eugen Rempel, Jörg Rahnenführer, Jan Hengstler and many colleagues for valuable contributions and insightful dis cussions. This work was supported by grants and support from the Doerenkamp Zbinden foundation, the German Research Foundation (RTG 1331) and the European Community's Sev enth Framework Programme (ESNATS project).

Conflict of interest

The authors declare no conflict of interests.

\section{References}

1 van Thriel C, Westerink RH, Beste C, Bale AS, Lein PJ, Leist M. Translating neurobehavioural endpoints of developmental neurotox icity tests into in vitro assays and readouts. Neurotoxicology 2012;33:911 24.

2 Grandjean P, Bellinger D, Bergman A, Cordier S, Davey Smith G, Eskenazi B et al. The faroes statement: human health effects of developmental exposure to chemicals in our environment. Basic Clin Pharmacol Toxicol 2008;102:73 5.

3 Meganathan K, Jagtap S, Wagh V, Winkler J, Gaspar JA, Hilde brand $\mathrm{D}$ et al. Identification of thalidomide specific transcriptomics and proteomics signatures during differentiation of human embry onic stem cells. PLoS One 2012;7:e44228.

4 Gassmann K, Abel J, Bothe H, Haarmann Stemmann T, Merk HF, Quasthoff KN et al. Species specific differential ahr expression protects human neural progenitor cells against developmental neu rotoxicity of pahs. Environ Health Perspect 2010;118:1571 7.

5 Bal Price AK, Coecke S, Costa L, Crofton KM, Fritsche E, Gold berg A et al. Advancing the science of developmental neurotoxic ity (dnt): testing for better safety evaluation. ALTEX 2012;29:202 15.

6 Adler S, Basketter D, Creton S, Pelkonen O, van Benthem J, Zuang $\mathrm{V}$ et al. Alternative (non animal) methods for cosmetics testing: current status and future prospects 2010. Arch Toxicol 2011;85:367 485 .

7 Basketter DA, Clewell H, Kimber I, Rossi A, Blaauboer B, Burrier $\mathrm{R}$ et al. A roadmap for the development of alternative (non animal) methods for systemic toxicity testing t4 report*. ALTEX 2012;29:3 91.

8 Klaassen C. Casarett and Doull's Toxicology: The Basic Science of Poisons, 7th edn. McGraw Hill, New York, 2010.

9 Kadereit S, Zimmer B, van Thriel C, Hengstler JG, Leist M. Com pound selection for in vitro modeling of developmental neurotoxic ity. Front Biosci 2012;17:2442 60 .

10 Kuegler PB, Zimmer B, Waldmann T, Baudis B, Ilmjarv S, Hesch eler $\mathrm{J}$ et al. Markers of murine embryonic and neural stem cells, neurons and astrocytes: reference points for developmental neuro toxicity testing. ALTEX 2010;27:17 42. 
11 Makris SL, Raffaele K, Allen S, Bowers WJ, Hass U, Alleva E et al. A retrospective performance assessment of the developmental neurotoxicity study in support of oecd test guideline 426. Environ Health Perspect 2009;117:17 25.

12 Lewis DA, Levitt P. Schizophrenia as a disorder of neurodevelop ment. Annu Rev Neurosci 2002;25:409 32.

13 Schwartzer JJ, Koenig CM, Berman RF. Using mouse models of aut ism spectrum disorders to study the neurotoxicology of gene envi ronment interactions. Neurotoxicol Teratol 2013;36:17 35.

14 Leist M, Hasiwa N, Daneshian M, Hartung T. Validation and qual ity control of replacement alternatives current status and future challenges. Toxicol Res 2012;1:8 22.

15 Leist M, Hartung T, Nicotera P. The dawning of a new age of tox icology. ALTEX 2008;25:103 14.

16 Hanson MA, Gluckman PD. Developmental origins of health and disease: new insights. Basic Clin Pharmacol Toxicol 2008;102:90 3.

17 Bihaqi SW, Zawia NH. Alzheimer's disease biomarkers and epige netic intermediates following exposure to pb in vitro. Curr Alzhei mer Res 2012;9:555 62.

18 Wu J, Basha MR, Brock B, Cox DP, Cardozo Pelaez F, McPher son CA et al. Alzheimer's disease (ad) like pathology in aged monkeys after infantile exposure to environmental metal lead $(\mathrm{pb})$ : evidence for a developmental origin and environmental link for ad. J Neurosci 2008;28:3 9.

19 Balmer NV, Weng MK, Zimmer B, Ivanova VN, Chambers SM, Nikolaeva E et al. Epigenetic changes and disturbed neural devel opment in a human embryonic stem cell based model relating to the fetal valproate syndrome. Hum Mol Genet 2012;21:4104 14 .

20 Ceccatelli S, Bose R, Edoff K, Onishchenko N, Spulber S. Long lasting neurotoxic effects of exposure to methylmercury during development. J Intern Med 2013;273:490 7.

21 Gilbert SF. Developmental Biology, 8th edn. Sinauer Associates Inc., Sunderland, MA, 2006.

22 Leist M, Bremer S, Brundin P, Hescheler J, Kirkeby A, Krause $\mathrm{KH}$ et al. The biological and ethical basis of the use of human embryonic stem cells for in vitro test systems or cell therapy. ALTEX 2008;25:163 90

23 Chazaud C, Yamanaka Y, Pawson T, Rossant J. Early lineage seg regation between epiblast and primitive endoderm in mouse blast ocysts through the grb2 mapk pathway. Dev Cell 2006;10:615 24.

24 Hass U. The need for developmental neurotoxicity studies in risk assessment for developmental toxicity. Reprod Toxicol 2006;22: 14856.

25 National Research Council. Scientific Frontiers in Developmental Toxicology and Risk Assessment, ed. Press NA, National Acad emy Press, Washington, DC, 2000.

26 Osmond C, Barker DJ. Fetal, infant, and childhood growth are pre dictors of coronary heart disease, diabetes, and hypertension in adult men and women. Environ Health Perspect 2000;108(Suppl. 3):545 53

27 Landrigan PJ, Sonawane B, Butler RN, Trasande L, Callan R, Droller D. Early environmental origins of neurodegenerative dis ease in later life. Environ Health Perspect 2005;113:1230 3.

28 Grandjean P. Late insights into early origins of disease. Basic Clin Pharmacol Toxicol 2008;102:94 9.

29 Zimmer B, Schildknecht S, Kuegler PB, Tanavde V, Kadereit S, Leist M. Sensitivity of dopaminergic neuron differentiation from stem cells to chronic low dose methylmercury exposure. Toxicol Sci 2011;121:357 67.

30 Stiegler NV, Krug AK, Matt F, Leist M. Assessment of chemical induced impairment of human neurite outgrowth by multiparamet ric live cell imaging in high density cultures. Toxicol Sci 2011;121:73 87.

31 Krug AK, Balmer NV, Matt F, Schoneberger F, Merhof D, Leist M. Evaluation of a human neurite growth assay as specific screen for developmental neurotoxicants. Arch Toxicol 2013;87:2215 31 .
32 Cory Slechta DA, Virgolini MB, Rossi George A, Thiruchelvam $\mathrm{M}$, Lisek R, Weston D. Lifetime consequences of combined mater nal lead and stress. Basic Clin Pharmacol Toxicol 2008;102:218 27.

33 Vahter M. Health effects of early life exposure to arsenic. Basic Clin Pharmacol Toxicol 2008;102:204 11.

34 Onishchenko N, Tamm C, Vahter M, Hokfelt T, Johnson JA, Johnson DA et al. Developmental exposure to methylmercury alters learning and induces depression like behavior in male mice. Toxicol Sci 2007:97:428 37.

35 Anway MD, Leathers C, Skinner MK. Endocrine disruptor vincloz olin induced epigenetic transgenerational adult onset disease. Endo crinology 2006;147:5515 23.

36 Skinner MK, Manikkam M, Guerrero Bosagna C. Epigenetic trans generational actions of environmental factors in disease etiology. Trends Endocrinol Metab 2010;21:214 22.

37 Skinner MK. What is an epigenetic transgenerational phenotype? F3 or f2. Reprod Toxicol 2008;25:2 6 .

38 Dulac C. Brain function and chromatin plasticity. Nature 2010;465:728 35.

39 Coppieters N, Dragunow M. Epigenetics in alzheimer's disease: a focus on DNA modifications. Curr Pharm Des 2011;17:3398 412.

40 Waldmann T, Schneider R. Targeting histone modifications epige netics in cancer. Curr Opin Cell Biol 2013;25:1849.

41 Zheng C, Hayes JJ. Structures and interactions of the core histone tail domains. Biopolymers 2003;68:539 46.

42 Jenuwein T, Allis CD. Translating the histone code. Science 2001;293:1074 80

43 Bannister AJ, Kouzarides T. Regulation of chromatin by histone modifications. Cell Res 2011;21:381 95 .

44 Smirnova L, Sittka A, Luch A. On the role of low dose effects and epigenetics in toxicology. EXS 2012;101:499 550.

45 Weng MK, Zimmer B, Poltl D, Broeg MP, Ivanova V, Gaspar JA et al. Extensive transcriptional regulation of chromatin modifiers during human neurodevelopment. PLoS One 2012;7:e36708.

46 Collotta M, Bertazzi PA, Bollati V. Epigenetics and pesticides. Toxicology 2013;307:35 41.

47 Stoccoro A, Karlsson HL, Coppede F, Migliore L. Epigenetic effects of nano sized materials. Toxicology 2013;313:3 14.

48 Fraga MF, Ballestar E, Paz MF, Ropero S, Setien F, Ballestar ML et al. Epigenetic differences arise during the lifetime of monozy gotic twins. Proc Natl Acad Sci USA 2005;102:10604 9.

49 Ramocki MB, Zoghbi HY. Failure of neuronal homeostasis results in common neuropsychiatric phenotypes. Nature 2008;455:912 8 .

50 Kramer JM, van Bokhoven H. Genetic and epigenetic defects in mental retardation. Int J Biochem Cell Biol 2009;41:96 107.

51 Graff J, Mansuy IM. Epigenetic dysregulation in cognitive disor ders. Eur J Neurosci 2009;30:1 8.

52 Amir RE, Van den Veyver IB, Wan M, Tran CQ, Francke U, Zo ghbi HY. Rett syndrome is caused by mutations in $\mathrm{x}$ linked mecp2, encoding methyl cpg binding protein 2. Nat Genet 1999;23:185 8

53 Murphy SK, Jirtle RL. Imprinted genes as potential genetic and epigenetic toxicologic targets. Environ Health Perspect 2000;108 (Suppl 1):5 11.

54 Weaver IC, Cervoni N, Champagne FA, D'Alessio AC, Sharma S, Seckl JR et al. Epigenetic programming by maternal behavior. Nat Neurosci 2004;7:847 54.

55 McGowan PO, Sasaki A, D'Alessio AC, Dymov S, Labonte B, Szyf $\mathrm{M}$ et al. Epigenetic regulation of the glucocorticoid receptor in human brain associates with childhood abuse. Nat Neurosci 2009;12:342 8.

56 Radtke KM, Ruf M, Gunter HM, Dohrmann K, Schauer M, Meyer A et al. Transgenerational impact of intimate partner violence on methylation in the promoter of the glucocorticoid receptor. Transl Psychiatry 2011;1:e21. 
57 Onishchenko N, Karpova N, Sabri F, Castren E, Ceccatelli S. Long lasting depression like behavior and epigenetic changes of bdnf gene expression induced by perinatal exposure to methylmer cury. J Neurochem 2008;106:1378 87.

58 Graff J, Rei D, Guan JS, Wang WY, Seo J, Hennig KM et al. An epigenetic blockade of cognitive functions in the neurodegenerat ing brain. Nature 2012;483:222 6 .

59 Peleg S, Sananbenesi F, Zovoilis A, Burkhardt S, Bahari Javan S, Agis Balboa RC et al. Altered histone acetylation is associated with age dependent memory impairment in mice. Science 2010;328:753 6 .

60 Skinner MK, Anway MD, Savenkova MI, Gore AC, Crews D. Transgenerational epigenetic programming of the brain transcrip tome and anxiety behavior. PLoS One 2008;3:e3745.

61 Crews D, Gillette R, Scarpino SV, Manikkam M, Savenkova MI, Skinner MK. Epigenetic transgenerational inheritance of altered stress responses. Proc Natl Acad Sci USA 2012;109: 91438.

62 Hartung T, McBride M. Food for thought. On mapping the human toxome. ALTEX 2011;28:83 93.

63 Blaauboer BJ, Boekelheide K, Clewell HJ, Daneshian M, Dinge mans MM, Goldberg AM et al. The use of biomarkers of toxicity for integrating in vitro hazard estimates into risk assessment for humans. ALTEX 2012;29:411 25.

64 Crofton KM, Mundy WR, Lein PJ, Bal Price A, Coecke S, Seiler $\mathrm{AE}$ et al. Developmental neurotoxicity testing: recommendations for developing alternative methods for the screening and prioritiza tion of chemicals. ALTEX 2011;28:9 15.

65 Saxena S, Caroni P. Mechanisms of axon degeneration: from development to disease. Prog Neurobiol 2007;83:17491.

66 Zikopoulos B, Barbas H. Changes in prefrontal axons may disrupt the network in autism. J Neurosci 2010;30:14595 609 .

67 Hussman JP, Chung RH, Griswold AJ, Jaworski JM, Salyakina D, Ma D et al. A noise reduction gwas analysis implicates altered regulation of neurite outgrowth and guidance in autism. Mol Aut ism 2011;2:1.

68 Harrill JA, Robinette BL, Freudenrich T, Mundy WR. Use of high content image analyses to detect chemical mediated effects on neu rite sub populations in primary rat cortical neurons. Neurotoxicolo gy 2013;34:61 73 .

69 Crofton KM, Mundy WR, Shafer TJ. Developmental neurotoxic ity testing: a path forward. Congenit Anom (Kyoto) 2012;52 1406.

70 Harrill JA, Robinette BL, Mundy WR. Use of high content image analysis to detect chemical induced changes in synaptogenesis in vitro. Toxicol In Vitro 2011;25:368 87.

71 Harrill JA, Freudenrich TM, Machacek DW, Stice SL, Mundy WR. Quantitative assessment of neurite outgrowth in human embryonic stem cell derived hn2 cells using automated high con tent image analysis. Neurotoxicology 2010;31:277 90.

72 Leist M, Hartung T. Inflammatory findings on species extrapola tions: humans are definitely no $70 \mathrm{~kg}$ mice. Arch Toxicol 2013;87:563 7 .
73 Tonk EC, Robinson JF, Verhoef A, Theunissen PT, Pennings JL, Piersma AH. Valproic acid induced gene expression responses in rat whole embryo culture and comparison across in vitro develop mental and non developmental models. Reprod Toxicol 2013;41:57 66.

74 Theunissen PT, Pennings JL, van Dartel DA, Robinson JF, Klein jans JC, Piersma AH. Complementary detection of embryotoxic properties of substances in the neural and cardiac embryonic stem cell tests. Toxicol Sci 2013;132:118 30.

75 Theunissen PT, Robinson JF, Pennings JL, de Jong E, Claessen SM, Kleinjans JC et al. Transcriptomic concentration response evaluation of valproic acid, cyproconazole, and hexaconazole in the neural embryonic stem cell test (estn). Toxicol Sci 2012;125:430 8 .

76 Zimmer B, Kuegler PB, Baudis B, Genewsky A, Tanavde V, Koh $\mathrm{W}$ et al. Coordinated waves of gene expression during neuronal differentiation of embryonic stem cells as basis for novel approaches to developmental neurotoxicity testing. Cell Death Dif fer 2011;18:383 95.

77 Zimmer B, Lee G, Stiegler NV, Meganathan K, Sachinidis A, Stu der L et al. Evaluation of developmental toxicants and signaling pathways in a functional test based on the migration of human neural crest cells. Environ Health Perspect 2012;120:1116 22.

78 Krug AK, Kolde R, Gaspar JA, Rempel E, Balmer NV, Megana than $\mathrm{K}$ et al. Human embryonic stem cell derived test systems for developmental neurotoxicity: a transcriptomics approach. Arch Toxicol 2013;87:123 43.

79 Hogberg HT, Kinsner Ovaskainen A, Coecke S, Hartung T, Bal Price AK. Mrna expression is a relevant tool to identify develop mental neurotoxicants using an in vitro approach. Toxicol Sci 2010;113:95 115.

80 Pallocca G, Fabbri M, Sacco MG, Gribaldo L, Pamies D, Laurenza I et al. Mirna expression profiling in a human stem cell based model as a tool for developmental neurotoxicity testing. Cell Biol Toxicol 2013;29:239 57.

81 Bal Price AK, Hogberg HT, Buzanska L, Lenas P, van Vliet E, Hartung T. In vitro developmental neurotoxicity (dnt) testing: rele vant models and endpoints. Neurotoxicology 2010;31:545 54.

82 Zimmer B, Lee G, Balmer NV, Meganathan K, Sachinidis A, Stu der L et al. Evaluation of developmental toxicants and signaling pathways in a functional test based on the migration of human neural crest cells. Environ Health Perspect 2012;120:1116 22.

83 Skinner MK, Manikkam M, Guerrero Bosagna C. Epigenetic trans generational actions of endocrine disruptors. Reprod Toxicol 2011;31:337 43 .

84 Senut MC, Cingolani P, Sen A, Kruger A, Shaik A, Hirsch H et al. Epigenetics of early life lead exposure and effects on brain development. Epigenomics 2012;4:665 74.

85 Bose R, Onishchenko N, Edoff K, Janson Lang AM, Ceccatelli S. Inherited effects of low dose exposure to methylmercury in neural stem cells. Toxicol Sci 2012;130:383 90. 